\title{
Open Trial of an Adaptation of Cognitive Processing Therapy for Message-Based Delivery
}

\author{
Shannon Wiltsey Stirman ${ }^{1,2}$, Jiyoung Song ${ }^{1}$, Thomas D. Hull ${ }^{3,4}$, and Patricia A. Resick ${ }^{5}$ \\ ${ }^{1}$ Dissemination and Training Division, National Center for PTSD, VA Palo Alto Health Care System, Menlo Park, California, \\ United States \\ ${ }^{2}$ Department of Psychiatry and Behavioral Sciences, Stanford University \\ ${ }^{3}$ Department of Counseling and Clinical Psychology, Columbia University \\ ${ }^{4}$ Talkspace, New York, New York, United States \\ ${ }^{5}$ Department of Psychiatry and Behavioral Sciences, Duke Health
}

\begin{abstract}
Cognitive Processing Therapy (CPT) has been found to be effective in individual and group therapy formats for Posttraumatic Stress Disorder (PTSD). However, many people in need of evidence-based PTSD treatment do not receive therapy due to distance from or availability of CPT-trained therapists, work and family obligations, and other barriers to in-person treatment. One way to expand the reach of CPT is through an asynchronous messaging format. To assess feasibility and preliminary effectiveness, CPT-Text was developed and pilot tested in an open trial $(N=28)$ and then compared to a matched comparison sample of people receiving messaging therapy as usual (TAU; $n=23$ ) for PTSD through the same platform. The CPT-Text completion rate $(63 \%)$ was comparable to face-to-face and telehealth-delivered CPT in previous studies. Word count and use of video meetings were comparable or lower than TAU, suggesting feasibility of delivery of a structured, trauma-focused intervention in a texting format. CPT-Text resulted in improvement in both PTSD and depression in fewer days than required for clinically significant change in the TAU sample. This effect for PTSD was greater than for TAU and the pre-post and between-conditions effect size for change was large. This first examination of a structured, trauma-focused intervention delivered through a messaging therapy platform suggests that with further testing and refinement, asynchronous CPT delivered via text may be a feasible, efficient, and effective PTSD intervention.
\end{abstract}

Keywords: cognitive processing therapy, treatment access, telepsychotherapy, technology, PTSD

Multiple well-designed randomized controlled trials (RCTs) have established efficacy for Cognitive Processing Therapy (CPT) with individuals from a number of backgrounds, including people who experienced rape, interpersonal violence, or other traumatic events, active duty service members, and veterans with posttraumatic stress disorder (PTSD; Bass et al., 2013; Butollo et al., 2016; Forbes et al., 2012; Resick et al., 2008, 2017). Additional uncontrolled research has demonstrated that individuals in the community, including refugees and Latinx populations, experience considerable symptom improvement when they receive CPT (LoSavio et al., 2019; Marques et al., 2019; Schulz et al., 2006).
Engagement and access to evidence-based treatments (EBTs), however, have remained low in most healthcare settings. Only $20 \%$ of the eligible Veterans Health Administration patient population and less than $25 \%$ of the Department of Defense service members with PTSD receive an EBT (Maguen et al., 2019; Wilk et al., 2013). Accessing EBTs for PTSD can be challenging due to availability of trained local therapists and barriers related to seeking any form of therapy: transportation, cost, work and family obligations, and stigma (Alleman, 2002; Hollon et al., 2002; Nutting et al., 2002; Young, 2005). Traditional psychotherapy often requires attending sessions during the workday. Travel to a therapist office or clinic for
Action Editor: Danielle S. McNamara was the action editor for this article. ORCID iD: Shannon Wiltsey Stirman (DD https://orcid.org/0000-00019917-5078; Jiyoung Song (D) https://orcid.org/0000-0002-7157-8198; Thomas D. Hull (D) https://orcid.org/0000-0001-8275-6765; Patricia A. Resick (D) https://orcid.org/0000-0002-2203-2404

Our data will be shared with researchers at request for the purpose of verifying published findings through reanalysis.

Acknowledgments: Preliminary data with a partial dataset were presented at the American Psychological Association and the American Psychiatric Association. We gratefully acknowledge the therapists for this study: Jennifer Wachen, PhD, Stefanie LoSavio, PhD, Mary Beth Shea, PhD, Loree Siegfried, CSW, Charity Wilkinson, PhD, John Moring, $\mathrm{PhD}$, Shareen Kneeshaw, PhD, Jane Higham, PhD., Patricia Bennett, PhD, and Kristy Morris, PhD. Mr. Song is now a graduate student in the Department of Psychology at the University of California, Berkeley.

Conflict of Interest Statement: The senior author was funded by Talkspace (pilot funding awarded to Duke Health) and Duke Health.
The first author received an honorarium from pilot study funds to write the therapist CPT-Text manual. The second author has no conflicts of interest to disclose. The third author is an employee of Talkspace, the platform used to deliver the messaging therapy, and has a financial interest in the company.

Disclaimer: Interactive content is included in the online version of this article.

Open Access License: This work is licensed under a Creative Commons Attribution-NonCommercial-NoDerivatives 4.0 International License (CC-BY$\mathrm{NC}-\mathrm{ND}$ ). This license permits copying and redistributing the work in any medium or format for noncommercial use provided the original authors and source are credited and a link to the license is included in attribution. No derivative works are permitted under this license.

Contact Information: Correspondence concerning this article should be addressed to Shannon Wiltsey-Stirman, Dissemination and Training Division, National Center for PTSD, VA Palo Alto Health Care System, 795 Willow Road (NC-PTSD 334), Menlo Park, CA 94025, United States. Email: sws1@stanford.edu 
a 50-min appointment weekly over several months can be challenging. Other individuals do not seek face-to-face treatment due to stigma or time constraints related to work and family obligations that preclude regular therapy attendance. Still others may have experienced thwarted attempts to engage in face-to-face care due to waitlists or insufficient insurance coverage. These challenges are even more pronounced when individuals seek specific EBTs in many areas of the country, as there are fewer providers with training in these interventions. For example, in some states, there is only one rostered community-based CPT provider (Cognitive Processing Therapy [CPT], 2020). Even when individuals are able to find trained local providers, those providers may have full caseloads or not accept insurance. While trauma-focused PTSD treatments have been shown to be effective when delivered by video conferencing (Kuhn \& Owen, 2020; Maieritsch et al., 2016; Morland et al., 2015), barriers to treatment delivery remain in more remote areas where individuals have limited access to internet service. Furthermore, telehealth, like in-person therapy, typically requires individuals to participate in prescheduled treatment sessions during the workday.

Online and mobile app interventions for PTSD are alternatives to traditional psychotherapy and telehealth that can address some of these barriers. Reviews and meta analyses have demonstrated the effectiveness of online and mobile app interventions for PTSD, particularly trauma-focused and therapist-guided interventions (Kuhn \& Owen, 2020; Kuester et al., 2016; Melville et al., 2010). However, as is the case with psychotherapy, many consumers do not sustain their engagement in these interventions (Kuhn \& Owen, 2020). Thus, there is a considerable need to identify additional efficient, acceptable, and effective forms of care, particularly for those who are less likely to access traditional psychotherapy or use online programs.

One potential solution to the above treatment barriers is asynchronous texting therapy. Smartphone ownership is common, and texting has become a preferred method of communication for many adults (Atun \& Sittampalam, 2006; Rice \& Katz, 2003). Mental health treatment via text messaging has become more common in recent years, with several commercial companies offering this service (Hoermann et al., 2017; Lindhiem et al., 2015). Synchronous text-based therapy has been shown to be more effective than wait list controls and equivalent, but not superior, to in person therapy (Hoermann et al., 2017). Studies have shown that as an adjunct to face-to-face cognitive behavioral therapy, messaging is associated with greater retention in psychotherapy (Aguilera et al., 2017). Some messaging therapy platforms offer asynchronous communication, which does not require participants to be present and respond to one another at the same time. Such platforms may facilitate engagement in treatment among those who seek discrete and affordable support, at times that are convenient and not disruptive to their busy schedules (Hull et al., 2020). Given the effectiveness of texting in engaging and retaining clients in psychotherapy, asynchronous texting therapy delivered by experienced therapists thus might increase client access to and engagement in EBTs.

While somewhat less is known about the effectiveness of asynchronous texting therapy, a small study demonstrated evidence of significant symptom change in nearly half of the individuals who sought treatment for a variety of presenting problems (Hull \& Mahan, 2017). In more recent asynchronous texting studies with larger samples, $66 \%$ of the clients who received treatment for depression and anxiety reported significant symptom change (Hull et al., 2020), and nearly half of the individuals who sought treatment for PTSD experienced clinically significant improvement (Malgaroli et al., 2020). In the latter study, the therapy modalities used to address PTSD were at the discretion of the therapist, and individuals could engage with their therapists 5 days per week through video messaging or texting. To date, however, there have not been studies to examine feasibility or effectiveness of a more structured EBT for PTSD in an asynchronous messaging format.

Our primary goal was to conduct a preliminary examination of feasibility, engagement, and symptom change among individuals who sought treatment for PTSD on a messaging therapy platform and agreed to participate in a non-randomized pilot evaluation of messaging-based Cognitive Process Therapy (CPT-Text). We define feasibility as the extent to which CPT could be successfully carried out in a texting format. Evidence of feasibility includes therapist delivery of modules specified by the protocol using similar or lower word count than Messaging Therapy as Usual (TAU), as well as reasons for dropout (e.g., technical difficulties related to CPT-Text). To examine engagement, defined as the extent to which clients participated in CPT-Text, we examined enrollment (compared to TAU), dropout rates, word generation, and time to completion of the protocol. We also conducted a non-randomized comparison of PTSD symptom change among individuals who engaged in CPT-Text with that of a matched cohort who received TAU. We hypothesized that clients who engaged in CPT-Text would experience significant and clinically meaningful improvement in PTSD symptoms. Because it is a trauma-focused intervention based on an EBT, we further hypothesized that CPT-Text would result in significantly greater symptom change than TAU.

\section{Method}

\section{Participants}

\section{Recruitment and Enrollment}

Talkspace advertises its services in print, online, television, and on social media. Some ads offered CPT-Text free of charge and some participants learned about the option through their intake process or Talkspace therapist after responding to a standard Talkspace ad or referral. Talkspace staff assessed interested individuals using Talkspace's standardized screening and intake procedures to determine a trauma-related presenting complaint and suitability for the platform. Eligible individuals were those residing in the United States who were assessed at their Talkspace intake call to have probable PTSD (and who scored a 33 or above on the PTSD Checklist-5; Blevins et al., 2015) and were not at high enough risk for suicidal thoughts and/or behavior to be marked a "Yes" on any of questions three through six on the Columbia Suicide Severity Rating Scale Lifetime-Recent Screen (Oquendo et al., 2003). During the intake meeting with a Talkspace therapist, clients were screened for psychosis and substance abuse that required prioritization in treatment and/or a higher level of care, and none endorsed or presented with these conditions. Individuals who were eligible and consented to participate in CPT-Text were placed with a CPTtrained therapist who was licensed in the client's state and created an account on the platform. During sign-up for the platform, individuals are routinely consented to the use of platform data in 
a de-identified and anonymous format for research purposes. University institutional review boards determined that the protocol qualified for exempt status.

\section{Messaging-Based Cognitive Processing Therapy}

One hundred and one individuals were screened, and reasons for non-inclusion were (a) no therapist in their state $(n=12)$ and (b) baseline score below the cutoff for probable PTSD $(n=29)$. Sixty individuals met inclusion criteria and were assigned a CPT-Text therapist, but 32 individuals did not subsequently enroll by creating Talkspace accounts in order to begin the texting intervention, resulting in a $47 \%$ enrollment rate. The sample of participants $(N=28)$ who participated in CPT-Text were $84 \%$ Female, $86 \%$ White, $50 \%$ in the age range of $26-35$. Forty-seven percent were married, and $73 \%$ of them had a bachelor's degree or higher. Based on the Life Events Checklist-5 (LEC-5; Weathers et al., 2013b), 13 participants reported sexual assault as their index trauma, five experienced assault with a weapon, three experienced the sudden violent death of a loved one, one experienced physical assault, and one experienced another unwanted or uncomfortable sexual experience. Eighty-two percent of the individuals who participated reported that they had experienced multiple traumatic events. Travel distance from the area in which participants lived to the office of closest CPTtrained therapist from the official roster (CPT, 2020) based on client zip codes ranged from 34 to 145 miles (70 miles on average). Three clients lived in a state with no CPT therapist (study therapists were licensed but did not have an office in the clients' states).

\section{Messaging Therapy as Usual}

We employed propensity score matching (Dehejia \& Wahba, $2002)$ to select clients in TAU $(n=23)$ who had similar baseline PTSD symptom severity profiles on the PTSD symptom clusters (Reexperiencing, Avoidance, Negative Affect, Arousal; Blevins et al., 2015) and number of days spent in treatment. Propensity score matching allowed us to select a comparison group (TAU) that was similar to the CPT-Text group in terms of symptom presentation, alleviating potential bias caused by systematic differences between the two conditions in a non-randomized comparison. In the current study, we used the nearest neighbor matching algorithm.

Characteristics of the matched comparison sample are shown in Table 1 . The 23 out of 28 clients in CPT-Text who reported PCL-5 scores at least twice were matched with those in TAU who were seen for similar timeframes and presented with similar baseline PTSD symptom profiles. Participants in TAU were $87 \%$ Female, $46 \%$ in the age range of $26-35$, and $39 \%$ married, and $68 \%$ of them had a bachelor's degree or higher. The enrollment rate for individuals who receive TAU through Talkspace is $12.5 \%$, much lower than CPT-Text (47\%).

\section{Measures \\ Post-Traumatic Stress Disorder Checklist for DSM-5}

The Post-traumatic Stress Disorder Checklist for DSM-5 (PCL-5; Weathers et al., 2013a) is a 20-item self-report questionnaire on PTSD symptoms from the Diagnostic and Statistical Manual of Mental Disorders (DSM-5; American Psychological Association [APA], 2013). Clients rated their PTSD symptom severity on a 5point Likert scale, from $0=$ not at all to $4=$ extremely at baseline and during treatment. The PCL-5 has demonstrated strong internal consistency in the literature, ranging from .94 to .96 (Blevins et al., 2015; Bovin et al., 2016). Individuals in CPT-Text completed the PCL-5 weekly. Individuals in TAU completed it once every 3 weeks, as is standard practice on the Talkspace platform.

\section{Patient Health Questionnaire-8}

In addition to the PCL-5, clients in CPT-Text also completed the eight-item depression scale weekly during their treatment, but not at their baseline assessment. Developed from the Patient Health Questionnaire-9 (Kroenke et al., 2001), the PHQ-8 assesses how frequently individuals experience depressive symptoms (excluding suicidal ideation) on a 4-point Likert scale, from $0=$ not at all to 3 = nearly every day. The PHQ-8 has demonstrated strong diagnostic reliability in the general population (Kroenke et al., 2009) and internal reliability of .88 (Shin et al., 2019). The PHQ- 8 was used for this pilot because it was used in Talkspace's routine clinical data collection procedure for depression at the time that the pilot began, and depression is routinely assessed during CPT and CPTText. However, it was not collected as a matter of routine for individuals seeking TAU for PTSD.

\section{Procedure}

\section{Messaging Platform}

The intervention was delivered on a digital mental health platform (Talkspace) that enables licensed therapists to provide treatment through asynchronous text message, audio and video messages, and live streaming video. It uses a HIPAA-compliant, secure messaging platform that allows individuals to receive care on their personal mobile device. The platform is routinely evaluated by external agencies to meet HITECH, SOC-2, and GDPR standards.

Table 1

Comparison of Baseline and Treatment Characteristics for CPT-Text and TAU

\begin{tabular}{lccccrr}
\hline \multicolumn{5}{c}{ PCL-5 symptom clusters } \\
\hline Treatment & PCL-5 Total & Reexperiencing & Avoidance & Negative affects & Arousal & Days in Tx \\
\hline CPT-Text & $52.17(14.52)$ & $12.39(3.73)$ & $6.52(1.56)$ & $19.65(6.05)$ & $13.61(6.09)$ & $56.78(34.54)$ \\
TAU & $51.96(11.60)$ & $12.52(3.69)$ & $6.70(1.40)$ & $19.26(5.28)$ & $13.48(4.82)$ & $73.00(88.54)$
\end{tabular}

Note. $n=23$ for each treatment. Numbers in parentheses represent standard deviation for the mean values. CPT-Text $=$ messaging-based Cognitive Processing Therapy; TAU = messaging therapy as usual; PCL-5 = PTSD Checklist-5. TAU sample was matched to CPT sample using propensity scores based on their total and cluster PCL-5 Scores and treatment duration. 


\section{Interventions}

Messaging-Based Cognitive Process Therapy. CPT-Text was based on the standard CPT protocol (Resick et al., 2016). CPT is a trauma-focused cognitive therapy in which individuals learn to examine and change problematic beliefs about their role in the trauma, themselves, and the world that were changed or were reinforced by traumatic events (Resick et al., 2008). It was originally designed to be 12 sessions, but more recent research has supported a flexible length of 6-18 sessions (Galovski et al., 2012). Both standard CPT and CPT-Text use a series of exercises and worksheets at every session to allow clients to practice the concepts and skills. In the first phase, the focus is on psychoeducation and identifying and differentiating thoughts and feelings. The middle phase of treatment focuses on trauma processing and cognitive restructuring skills. In the final phase of treatment, the cognitive skills are combined and applied to different beliefs that are commonly impacted through trauma: safety, trust, power and control, esteem, and intimacy.

The adaptation for messaging-based therapy (CPT-Text) included the same content as that provided in the face-to-face or video telehealth protocols for CPT without a written trauma account (Resick et al., 2016), delivered in the form of 12 modules. After clients were screened, consented to treatment, and onboarded to the Talkspace platform, therapists and clients had the option of having an initial 30-min video conference to facilitate engagement before beginning CPT-Text. CPT-Text uses a client manual and links to a standard set of animated whiteboard videos to convey, illustrate, and facilitate practice of key concepts and skills. Clients received a PDF workbook that contained the same psychoeducation information, handouts, and worksheets that therapists would typically provide in face-to-face sessions.

During CPT-Text, therapists could introduce new skills and modules whenever they determined that clients had sufficient practice or understanding of the previous concepts. At the appropriate points in the protocol, therapists sent links to the brief whiteboard videos (which were hosted on a secure webpage outside the Talkspace platform) that introduced new skills and concepts that would normally be covered verbally during face-to-face therapy. These videos also demonstrated how to complete different CPT exercises and skill worksheets. Therapists encouraged the client to review the video links and psychoeducation in the manual, complete exercises, and reflect on their beliefs. Clients completed assigned exercises and posted them to the platform. As is standard on the Talkspace platform, therapists reviewed each client's dashboard at two designated times per day, 5 days per week. They responded to client contacts and assignments with feedback and posed Socratic questions to assist them in examining their beliefs. In contrast to face-to-face treatment, in which Socratic questions build on clients' responses to previous questions, therapists often asked a series of Socratic questions in a single message, and clients posted responses at their convenience. Therapists and clients could engage in up to three additional 30-min video conferences if needed to improve engagement or to address challenging issues in a focused manner. Clients who completed all 12 of the CPT-Text modules were considered to have completed the entire protocol. Clients were given up to 18 weeks to complete the protocol at their own pace. If they discontinued CPT-Text before the end of the protocol or did not complete it by 18 weeks, they were offered standard Talkspace therapy or a list of local therapists. Consistent with the CPT protocol, clients who received CPT-Text completed a weekly PCL-5 to assess symptoms of PTSD and a weekly Patient-Health Questionnaire-8 (PHQ-8; Kroenke et al., 2009) to assess symptoms of depression.

We solicited CPT-Text therapists through an email to the full cohort of qualified CPT trainers and consultants from the official CPT provider roster (CPT, 2020), and one CPT-trained Talkspace therapist also volunteered. Ten PhD-level therapists and one master's level therapist were trained in the use of the messaging platform and provided with a manual for the CPT-Text adaptation that included guidance on delivering the protocol in a messaging format. There were several group calls over the course of the study, which focused on adapting CPT to a messaging format, and therapists were invited to contact the first author if they needed consultation. The first author reviewed a subset (30\%) of transcripts to assess fidelity and provided limited guidance on cases. Nine therapists ultimately began CPT-Text with one or more individuals, who lived in 12 different states. The maximum number of clients seen by a single therapist was five, and most therapists treated one to two clients.

Messaging Therapy as Usual. TAU was conducted with clients who enrolled in Talkspace through the platform's normal onboarding and intake processes. While therapists who utilize the platform report a variety of therapeutic approaches and areas of expertise (see Hull et al., 2020 for a full description of the therapy network), those therapists who endorsed a specialty in treating PTSD and who identified CBT as one of their orientations were selected for this study. Clients who were being treated by these clinicians primarily for their PTSD-related symptoms were selected. In all, eight therapists, treating three clients each on average, were included in the TAU cohort. Therapists were all master's level clinicians with 9.4 years $(S D=5.2)$ of post-licensure experience. Therapists communicated on the same asynchronous schedule, with at least two contacts per day, 5 days per week, and could engage in video therapy as frequently as desired. This further strengthened the similarity in treatment between the TAU and CPT-Text groups.

Therapists were free to use any modality or intervention they believed was appropriate to address the client's treatment needs and concerns. Examination of de-identified transcripts by a member of the research team indicated that as is often the case in usual face-toface therapy, treatment was less structured than CPT-Text. Therapists used an eclectic mix of therapeutic strategies, including checking in on how the client was feeling and commenting on the client's updates with supportive and person-centered approaches, some cognitive behavioral strategies, and suggesting resources and activities (e.g., books to read, journaling; See Table 2).

\section{Analytic Plan}

In the current study, we performed our statistical analyses in $\mathrm{R}$ (R Core Team, 2018) to model and compare symptom trajectories between CPT-Text and TAU. We used the lme4 (Bates et al., 2015) and lmerTest (Kuznetsova et al., 2017) packages for multilevel modeling and the MatchIt (Ho et al., 2011) package for propensity score matching. 


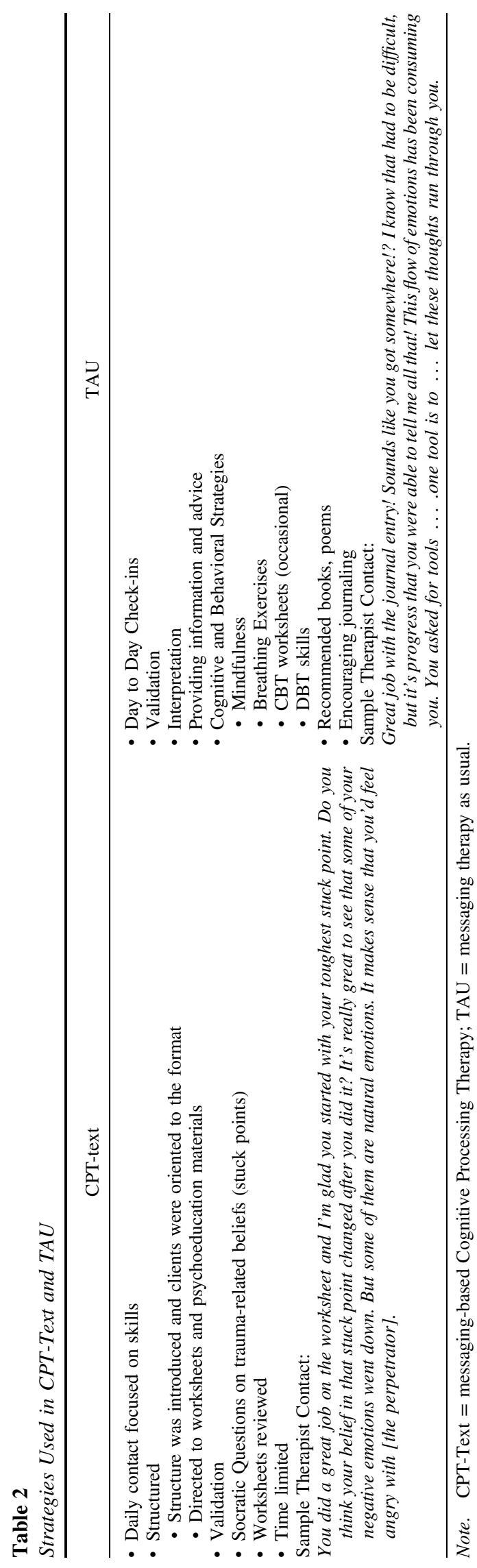

\section{Survival Analysis and Client Engagement}

To better understand clients' treatment engagement in CPT-Text, we conducted survival analysis. If clients stopped responding to their therapists on the platform before they had completed all 12 CPT-Text modules, we considered them to have dropped out. Those who completed all modules of the protocol were considered completers. We also examined word count for an estimate of engagement with the platform, as Talkspace's internal evaluation has shown word count to be a better approximation of engagement than logins. Word count estimates were not normally distributed and were therefore logtransformed before conducting a $t$-test to compare conditions. We estimated the clients' number of weeks spent in treatment based on the dates of their last weekly assessments or, for completers, their postcompletion assessment. To understand reasons for dropout and to distinguish dropout from early completion (Galovski et al., 2012; Szafranski et al., 2017), we examined CPT-Text transcripts to record reasons for non-completion if they were provided. We also examined the mean time clients stayed in treatment before their PCL-5 scores fell below the cutoff scores of 33 for probable PTSD and 20 for good endstate functioning (Wachen et al., 2019).

\section{Multilevel Modeling}

To examine symptom improvement for CPT-Text, we created longitudinal multilevel models. Because each therapist met with multiple clients, and each client reported multiple symptom measures, our data had the following nesting structure: repeated assessments (Level-1) nested within a client (Level-2). Given that there were a small number of clients per therapist in both conditions, we did not include the therapists as a nesting level in our models. We first included only the intercept as a predictor and evaluated the two-level unconditional models to examine the variance distribution of our symptom measures for each of our nesting level. In our longitudinal multilevel models, we allowed our initial symptom severity (random intercept) and time (number of days since each client's first assessment; random slope) to vary by the nesting levels. We also estimated the effect sizes of pre- to posttreatment changes with Cohen's $d$ (small $=0.20$, medium $=0.50$, large $=0.80$; Cohen, 1977) and fixed effects in multilevel regression with partial regression coefficients, $p r($ small $=.10$, medium $=.24$, large $=.37$; Kirk, 1996).

\section{Comparison to Messaging Therapy as Usual}

To compare the rates of PTSD symptom change between the two matched samples, we added the binary variable for treatment condition $(\mathrm{CPT}-\mathrm{Text}=1$, TAU $=0$ ) as a moderator to our time variable in longitudinal multilevel modeling. Because TAU is more open-ended in nature and did not follow a specific protocol, and clients were matched to CPT-Text in part based on treatment length. We did not examine dropout or reasons for dropout in the TAU sample.

\section{Results}

\section{Feasibility, Treatment Engagement, and Reasons for Dropout}

Twenty-eight clients began CPT-Text, and 18 (63\%) completed the protocol. Of the 10 who did not complete the protocol: Three 
decided to seek face to face therapy (one was able to access in-person CPT), three cited problems with technology (e.g., phone or computer with an older and unsupported operating system), one stated that they did not find the approach to be helpful, and one stopped due to health problems. Two did not respond after receiving the first psychoeducation video and did not provide a reason for discontinuation. On average, clients in CPT-Text remained engaged for 46.64 days $(S D=34.54)$. Table 3 and Figure 1 present a life table and survival analysis of clients' engagement in CPT-Text. Neither baseline PCL-5, $b=0.00, t(26)=-0.08, p=.935$, nor PHQ-8, $b=0.03, t(23)=0.19, p=.851$, predicted length of treatment. Because length of treatment was a matching variable, average duration in treatment was not significantly different between the two conditions, $t(44)=0.82, p=.418$.

To assess feasibility in terms of therapist time providing the treatment and client engagement in the two conditions, we also examined the number of words generated by therapists and clients, and we examined engagement in video conferencing. For CPTText, therapists wrote a mean of 9,036 words $(S D=8,297)$ and clients wrote 7,400 words $(S D=7,877)$. For comparison, mean word counts for TAU treatments were 17,451 words $(S D=15,188)$ by therapists and 28,766 words $(S D=51,168)$ by clients. Therapist word generation was significantly lower in CPT-Text, $t(44.5)=-2.06, p=.046$, and the lower rate of client word generation for CPT-text was also significant, $t(44)=-2.42$, $p=.020$. Nine people in TAU engaged in one video conferencing meeting, while 10 in CPT-text did (three engaged in two video conference meetings). Treatment condition was not significantly associated with the frequency of live video sessions, $b=0.15$, $S E=0.43, p=0.731$. These findings support feasibility of CPTText in terms of time spent on sending messages and using primarily messaging rather than video to convey content and support skill development.

Table 3

Life Table of Treatment Engagement in CPT-Text

\begin{tabular}{|c|c|c|c|c|c|}
\hline $\begin{array}{c}\text { Interval } \\
\text { between } \\
\text { weeks }\end{array}$ & $\begin{array}{c}\text { Number } \\
\text { in treat- } \\
\text { ment }\end{array}$ & $\begin{array}{l}\text { Number } \\
\text { who } \\
\text { dropped } \\
\text { out }\end{array}$ & $\begin{array}{l}\text { Proportion } \\
\text { who } \\
\text { dropped } \\
\text { out (ha- } \\
\text { zard) }\end{array}$ & $\begin{array}{l}\text { Proportion } \\
\text { remaining } \\
\text { in treat- } \\
\text { ment (sur- } \\
\text { vival) }\end{array}$ & $\begin{array}{l}\text { Cumulative } \\
\text { proportion } \\
\text { remaining } \\
\text { in treatment }\end{array}$ \\
\hline $1-2$ & 28 & 5 & .18 & .82 & .82 \\
\hline $2-3$ & 23 & 1 & .04 & .96 & .79 \\
\hline $3-4$ & 22 & 0 & .00 & 1.00 & .79 \\
\hline $4-5$ & 22 & 3 & .14 & .86 & .68 \\
\hline $5-6$ & 19 & 4 & .21 & .79 & .54 \\
\hline $6-7$ & 15 & 2 & .13 & .87 & .46 \\
\hline $7-8$ & 13 & 1 & .08 & .92 & .43 \\
\hline $8-9$ & 12 & 1 & .08 & .92 & .39 \\
\hline $9-10$ & 11 & 1 & .09 & .91 & .36 \\
\hline $10-11$ & 10 & 0 & .00 & 1.00 & .36 \\
\hline $11-12$ & 10 & 1 & .10 & .90 & .32 \\
\hline $12-13$ & 9 & 3 & .33 & .67 & .21 \\
\hline $13-14$ & 6 & 3 & .50 & .50 & .10 \\
\hline $14-15$ & 3 & 2 & .67 & .33 & .04 \\
\hline $15-16$ & 1 & 0 & .00 & 1.00 & .04 \\
\hline $16-17$ & 1 & 0 & .00 & 1.00 & .04 \\
\hline $17-18$ & 1 & 1 & 1.00 & .00 & .00 \\
\hline
\end{tabular}

Note. $\quad$ CPT-Text $=$ messaging-based Cognitive Processing Therapy.
Figure 1

Proportion of Clients Remaining in CPT-Text by the Beginning of Each Week

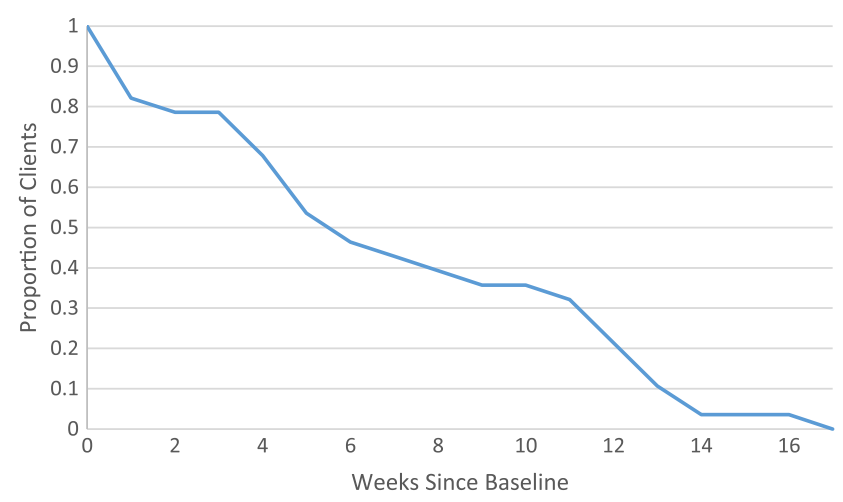

Note. $\quad$ CPT-Text $=$ messaging-based Cognitive Processing Therapy.

\section{PTSD Symptoms}

The mean baseline PCL-5 score for CPT-Text was 52.17 $(S D=14.52)$, and the posttreatment mean was $27.30(S D=$ 22.48). In the multilevel analysis, the unconditional model indicated that about $50 \%$ of the variance in PTSD symptoms was attributed to the repeated assessments over time in treatment (Level-1), and 50\% to clients (Level-2). Intent-to-treat analyses indicated that PTSD symptoms significantly improved over the course of CPT-Text, $b=-.45, t(13.64)=-6.38, p<.001, p r=-.87$; see Table 4. The mean reduction in PCL-5 scores from baseline to post-treatment was 24.87 points $(S D=19.27)$, a large treatment effect, $d=-1.29$, $95 \%$ CI $[-2.18,-0.37]$. Of the 23 people who completed the PCL-5 for at least two timepoints, $14(61 \%)$ reported reliable improvement of 17 or more points on the PCL-5 per Jacobson and Truax (1991). Among those 14,13 people $(57 \%)$ ended treatment with a PCL-5 score below 33 after on average 31.54 days $(S D=27.47)$, and nine individuals $(39 \%)$ ended with a PCL-5 score below 20 (considered to be a good end-state on the scale; Wachen et al., 2019) after an average of 46.78 days $(S D=20.33)$.

Of the 23 people in the matched TAU sample, 7 (30\%) reported reliable improvement of 17 or more points on the PCL5 (Jacobson \& Truax, 1991). Among those seven, five (22\%) ended treatment with a PCL-5 score below 33 after on average 123.8 days $(S D=115.37)$, and three $(13 \%)$ ended with a PCL-5 score below 20 after on average 160.67 days $(S D=140.41)$. Comparisons could not be made for the PHQ- 8 because this information is not routinely collected for individuals presenting with primary probable PTSD in TAU.

Our two-level unconditional model indicated that about $50 \%$ of the variance in the combined sample's PTSD symptoms was attributed to the repeated assessments within each client (Level-1), and 50\% to the clients (Level-2). Table 5 and Figure 2 demonstrate that clients significantly improved over time, $b=-0.19, t(23.93)=-2.68, p=.0130, p r=-.48$, regardless of their treatment conditions. However, clients in CPT-Text experienced more rapid improvements, $b=-0.27$, $t(22.62)=-2.88, p=.008, p r=-.52$. The mean reduction in PCL-5 scores from pre- to posttreatment was twice as large in CPT-Text $(M=24.87, S D=19.27)$ as TAU $(M=11.26$, 
Table 4

Fixed Effects of Longitudinal Multilevel Modeling of PCL-5 Scores in CPT-Text

\begin{tabular}{lcccccrr}
\hline & & & \multicolumn{2}{c}{$95 \% \mathrm{CI}$} & & \\
\multicolumn{1}{c}{ Fixed effect } & $b$ & $S E$ & LL & UL & $p$ & $p r$ \\
\hline Intercept & 51.38 & 2.78 & 45.84 & 56.89 & $<.001$ & .96 \\
Days since baseline & -0.45 & 0.07 & -0.60 & -0.31 & $<.001$ & -.87 \\
\hline
\end{tabular}

Note. PCL-5 scores of clients significantly decreased over the course of CPT-Text. PTSD $=$ Posttraumatic Stress Disorder; CPT-Text $=$ messagingbased Cognitive Processing Therapy; PCL-5 $=$ PTSD Checklist-5; $\mathrm{CI}=$ confidence interval; $\mathrm{LL}=$ lower limit; $\mathrm{UL}=$ upper limit; $p r=$ partial regression coefficient $($ small $=.10$, medium $=.24$, large $=.37$; Kirk, 1996).

$S D=12.50)$. The between-conditions effect size for change was large, $d=0.84,95 \%$ CI $[0.23,1.44]$; see Table 6 .

\section{Depressive Symptoms}

Among the 25 CPT-Text clients who completed the PHQ-8, our unconditional model indicated that about $37 \%$ of the variance in depressive symptoms was attributed to the repeated assessments over time (Level-1), and $63 \%$ to the clients (Level-2). Clients significantly improved in their comorbid depressive symptoms over the course of CPT, $b=-0.13, t(11.10)=-4.08, p=.002$, $p r=-.77$; see Table 7 and Figure 3. Average reduction in PHQ-8 scores from baseline $(M=13.30, S D=6.88)$ to posttreatment $(M=7.81, S D=7.64)$ was 5.88 points $(S D=5.98)$, a large treatment effect, $d=-0.98,95 \%$ CI [-2.01, 0.08].

Fifteen of the 25 clients $(60 \%)$ who completed a PHQ-8 reported a score of 10 or below (cutoff for current depression; Kroenke et al., $2009)$ after a mean treatment duration of 29.80 days $(S D=22.99)$. Of 16 clients who reported their PHQ- 8 scores at least twice, 8 (50\%) experienced a symptom reduction greater than five points, which is considered a reliable improvement for depression treatment (Kroenke \& Spitzer, 2002) after a mean treatment duration of 34.63 days $(S D=17.34)$. Because the PHQ-8 is not collected routinely for individuals in TAU who have a primary PTSD diagnosis, we could not compare changes in depression between CPT-Text and TAU.
Figure 2

Comparison of Predicted PCL-5 Score Trajectories Between CPTText and TAU

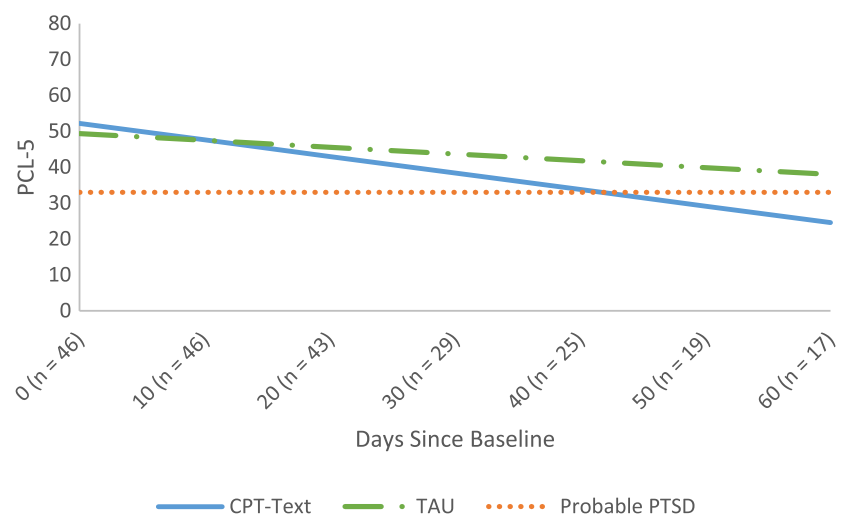

Note. On average, clients in CPT-Text experienced reductions in PTSD symptoms at a faster rate than those in TAU. Numbers in parentheses on the $\mathrm{x}$-axis represent the number of people remaining in either treatment. The horizontal line indicates the cutoff score of 33 for probable PTSD. PCL$5=$ PTSD Checklist-5; CPT-Text $=$ messaging-based Cognitive Processing Therapy; TAU $=$ messaging therapy as usual.

\section{Discussion}

We evaluated CPT-Text, an adaptation of CPT that was delivered through a novel message-based format, in a pilot study to assess feasibility, engagement, and preliminary effectiveness. Using several different indicators, we found that CPT-Text was feasible to implement. Individuals who were referred at intake or responded to ads for CPT were more likely to begin therapy than individuals who responded to ads for TAU. While the offer of free therapy likely contributed to the difference in enrollment, in combination with findings related to engagement and dropout, these findings also indicate that there were individuals who were open to engaging in a trauma-focused intervention over a messaging platform. Aside from not meeting criteria for probable PTSD, the largest reason for inclusion was not having a licensed CPT-Text therapist in their state. This feasibility issue could be addressed by training therapists in more states to provide CPT-Text if the intervention proves to be

\section{Table 5}

Fixed Effects of Longitudinal Multilevel Modeling Comparison of PCL-5 Scores Between CPT-Text and TAU

\begin{tabular}{|c|c|c|c|c|c|c|}
\hline \multirow[b]{2}{*}{ Fixed effect } & \multirow[b]{2}{*}{$b$} & \multirow[b]{2}{*}{$S E$} & \multicolumn{2}{|c|}{$95 \% \mathrm{CI}$} & \multirow[b]{2}{*}{$p$} & \multirow[b]{2}{*}{$p r$} \\
\hline & & & LL & UL & & \\
\hline \multicolumn{7}{|l|}{ Unconditional growth } \\
\hline Intercept & 51.01 & 1.91 & 47.22 & 54.77 & $<.001$ & .97 \\
\hline Days Since Baseline & -0.34 & 0.05 & -0.45 & -0.24 & $<.001$ & -.74 \\
\hline \multicolumn{7}{|l|}{ CPT-Text vs. TAU } \\
\hline Intercept & 49.38 & 2.73 & 43.95 & 54.67 & $<.001$ & .93 \\
\hline Days Since Baseline & -0.19 & 0.07 & -0.33 & -0.05 & .013 & -.48 \\
\hline CPT-Text & 2.80 & 3.81 & -4.62 & 10.32 & .467 & .11 \\
\hline Days Since Baseline $\times$ CPT-Text & -0.27 & 0.09 & -0.45 & -0.08 & .008 & -.52 \\
\hline
\end{tabular}

Note. The cross-level interaction term Days Since Baseline $\times$ CPT-Text indicates that PCL- 5 scores of clients in CPT-Text decreased at a significantly faster rate than those in TAU. PCL-5 = PTSD Checklist-5; CPT-Text = messaging-based Cognitive Processing Therapy; TAU = messaging therapy as usual; $\mathrm{CI}=$ confidence interval; $\mathrm{LL}=$ lower limit; $\mathrm{UL}=$ upper limit; $p r=$ partial regression coefficient $($ small $=.10$, medium $=.24$, large $=.37 ; \mathrm{Kirk}, 1996$ ). 
Table 6

Comparison of Average Reduction in PCL-5 Scores Between CPTText and TAU

\begin{tabular}{lcccccc}
\hline Treatment & $n$ & Change & $t$ & $d f$ & $p$ & $d$ \\
\hline CPT-Text & 23 & $24.87(19.27)$ & 2.84 & 37.73 & .007 & 0.84 \\
TAU & 23 & $11.26(12.50)$ & & & &
\end{tabular}

Note. From pre- to posttreatment, PCL-5 scores of CPT-Text clients decreased twice as much as those of TAU. PCL-5 = PTSD Checklist-5; CPTText $=$ messaging-based Cognitive Processing Therapy; TAU $=$ messaging therapy as usual; $d=$ Cohen's $d$ (small $=0.20, \quad$ medium $=0.50$, large $=0.80 ;$ Cohen, 1977).

effective in larger and more rigorous trials. Video sessions were not more frequent in CPT-Text than TAU, suggesting that the skill development and interactions required for CPT-Text could be successfully carried out over messaging without extensive reliance on video interactions. Additionally, word count was lower for CPTText, likely due to the use of whiteboard videos and worksheets to introduce and practice concepts. This limited the amount of text that therapists needed to generate for psychoeducation and introduction of specific skills, potentially increasing feasibility. Although time spent engaging in these activities for clients was not measured and word count did not include words generated on CPT worksheets that were assigned for practice, it appears these materials reduced the typing requirement for CPT-Text patients. In future studies, estimating time spent in CPT-Text and TAU, and assessing worksheet completion may also provide a more complete picture of feasibility and engagement.

The dropout rate for CPT-Text was similar to that of face-to-face CPT in some previous studies (Galovski et al., 2012; Resick et al., 2008) and somewhat lower than in studies of CPT via telehealth (Morland et al., 2015). These findings are consistent with reviews that have identified challenges in maintaining engagement in other forms of digital mental health intervention (Baumel et al., 2019; Torous et al., 2018). However, consistent with other findings (Lewis et al., 2019), the fact that a therapist checked in daily with clients may have supported greater engagement than is often seen for other forms of technology-based psychotherapy, such as mobile apps, which can be as low as 3\%-4\% for standalone apps and approximately $9 \%$ for apps that include peer support (Baumel et al., 2019). It will be useful in future investigations to address challenges related to technology and to refine CPT-Text to increase acceptability. Comparisons to lower intensity, self-guided digital interventions for

\section{Table 7}

Fixed Effects of Longitudinal Multilevel Modeling of PHQ-8 Scores in CPT-Text

\begin{tabular}{|c|c|c|c|c|c|c|}
\hline \multirow[b]{2}{*}{ Fixed effect } & \multirow[b]{2}{*}{$b$} & \multirow[b]{2}{*}{$S E$} & \multicolumn{2}{|c|}{$95 \% \mathrm{CI}$} & \multirow[b]{2}{*}{ D } & \multirow[b]{2}{*}{$p r$} \\
\hline & & & LL & UL & & \\
\hline Intercept & 15.51 & 1.20 & 13.05 & 17.88 & $<.001$ & .94 \\
\hline Days Since Baseline & -0.13 & 0.03 & -0.20 & -0.06 & .002 & -.77 \\
\hline
\end{tabular}

Note. PHQ-8 scores of clients significantly decreased over the course of CPT-Text. CPT-Text $=$ messaging-based Cognitive Processing Therapy; PHQ-8 = Patient Health Questionnaire-8; CI = confidence interval; $\mathrm{LL}=$ lower limit; $\mathrm{UL}=$ upper limit; $p r=$ partial regression coefficient $($ small $=.10$, medium $=.24$, large $=.37 ;$ Kirk, 1996).
Figure 3

Predicted PHQ-8 Score Trajectory of CPT-Text Clients

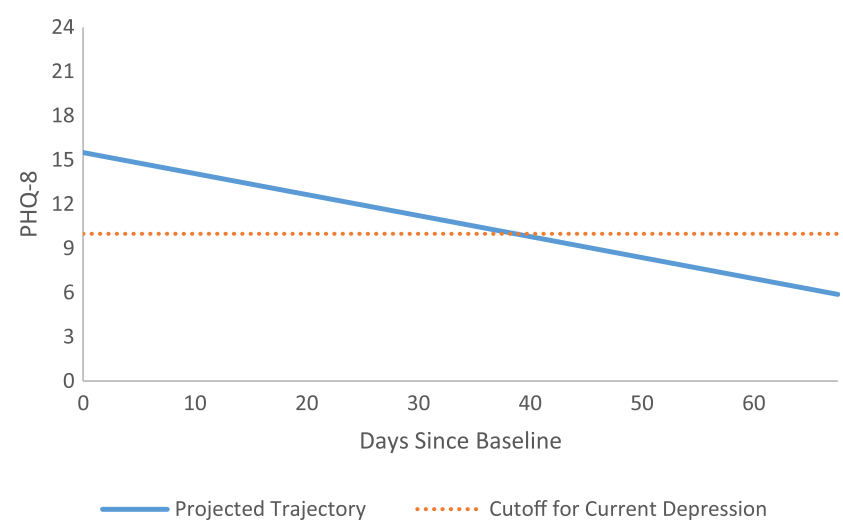

Note. The horizontal line indicates the cutoff score of 10 for current depression. PHQ-8 = Patient Health Questionnaire-8; CPT-Text = messaging-based Cognitive Processing Therapy.

PTSD as well as CPT provided through other modalities will also be useful in determining the population for whom CPT-text would be most beneficial and appropriate.

Among those who completed CPT-Text, most (78\%) experienced a clinically significant improvement in response to treatment, falling below the cutoff score for probable PTSD within about 3.5 weeks on average. The mean time to completion of the protocol for the CPT-text sample was briefer than the time required to complete a protocol of weekly face-to-face CPT sessions in routine care settings, which can range from 6 to 16 weeks (Galovski et al., 2012) but is typically more than 12 weeks (Monson et al., 2018). Neardaily contact and the ability to work through the content of an adaptation of an evidence-based protocol at one's own pace, rather than in one session per week, may allow individuals who are particularly motivated or who demonstrate a high level of understanding of CPT concepts and skills to experience rapid improvement. It may also allow for more timely support and feedback for individuals who may struggle to master the skills and concepts in a weekly therapy format. In fact, there was a large variation in the duration for treatment completion, reflecting variation in the pace with which clients were able to learn and use the skills with their therapists' guidance. Additionally, when compared to a sample that was matched in terms of symptom severity and time in treatment, individuals who participated in CPT-Text experienced significantly greater symptom change than TAU. Fewer individuals in TAU experienced clinically significant change or fell below the cutoff for probable PTSD during their time in treatment. Thus, while more rigorous study is necessary before drawing firm conclusions, this preliminary study suggests that CPT-Text may be a feasible, effective, and efficient strategy to address PTSD.

While CPT-Text demonstrated promising outcomes in this preliminary study, limitations are inherent in an evaluation of this nature. Our primary goal was to examine the feasibility, engagement, and preliminary effectiveness of CPT-Text. Therefore, we did not randomize individuals to CPT-Text or messaging as usual, and the study was not designed or adequately powered to detect differences. Consistent with the procedures used in routine texting therapy, we used self-report measures rather than diagnostic 
interviews to assess PTSD and depression symptoms. Additionally, the PHQ-8 prompts individuals to endorse symptoms over 2 weeks but in the CPT-Text sample, it was administered to individuals who received CPT-Text on a weekly basis, consistent with the frequency with which the PHQ-9 has been administered in other studies of texting-based assessment (Aguilera et al., 2015). Changes in depression could not be compared between CPT-Text and TAU because the PHQ- 8 was not collected routinely in usual care for PTSD, as is also the case in routine face-to-face therapy. Additionally, texting therapy as usual is not manualized and does not occur over a standardized period of time. Comparisons of dropout between manualized treatments and usual care are challenging, because many therapists do not systematically track unplanned terminations or disengagement from usual care (Baldwin et al., 2009). Thus, we could not conduct a direct comparison between messaging therapy formats for dropout. However, dropout rates (e.g., termination before therapeutic goals are achieved) in messaging as usual will be examined in future research. To overcome some of these limitations of the nonrandomized design, we employed propensity score matching based on baseline PTSD symptom profiles and treatment duration. We were, however, unable to include other matching variables, such as demographics, trauma history, types of index trauma, and comorbidity due to missingness in the archive data of the platform used for the TAU comparison. This limitation and the relatively low number of participating clients reduce the potential generalization of our findings to other populations. However, a matched comparison allowed us to examine the possible benefits of using more rigorous study designs in future comparisons of CPT-Text and messaging therapy as usual for PTSD.

It is also important to note that the majority of our sample were white, female, and college-educated. The lack of diversity might limit the generalizability of our results, because race and gender in particular play a role in exposure to and development of PTSD as well as treatment seeking behaviors (Roberts et al., 2011). However, the study make-up is similar to that reported elsewhere for the platform (Hull et al., 2020) and for the use of telehealth in more traditional therapy settings as well (Titov et al., 2017). Future research should address the limitations of this study by including a fully-powered randomized design, objective assessments, a more standardized comparison condition, a more diverse and larger sample, and systematic documentation of reasons for dropout. Additionally, factors such as user experience and therapeutic alliance should be examined (cf., Hull \& Mahan, 2017; Morland et al., 2015).

Although therapists in TAU had reported that they were experienced in treating clients with PTSD, it is possible that qualified CPT providers in the CPT-Text condition were more highly trained at treating PTSD. Therapist training (i.e., PhD vs. MA) is confounded with condition in this study and might have affected client treatment outcomes, although previous research has suggested that therapist degree is not predictive of clinical outcomes (Atkins \& Christensen, 2001). Nonetheless, if additional research confirms the effectiveness of this approach, we expect that future CPT-Text providers would undergo CPT training or training specifically in CPT-Text before implementing it with their clients online.

Digital mental health platforms have substantial potential to reduce barriers in accessing PTSD treatment. This was the first examination of a structured, trauma-focused intervention over a messaging therapy platform. Our findings were consistent with the growing literature that suggests that internet-based interventions for PTSD can be feasible and effective, particularly with therapist guidance (Kuhn \& Owen, 2020; Melville et al., 2010). This format may allow for discrete, efficient, and effective delivery of an adequate dose of treatment without requiring travel to in-person appointments during the workday. If future research with larger samples replicates or improves upon these findings, and particularly if strategies are identified to increase engagement or identify individuals for whom CPT-Text is a good fit, it may result in an accessible format for evidence-based PTSD treatment that addresses common barriers to care.

\section{References}

Aguilera, A., Bruehlman-Senecal, E., Demasi, O., \& Avila, P. (2017). Automated text messaging as an adjunct to cognitive behavioral therapy for depression: A clinical trial. Journal of Medical Internet Research, 19(5), e148. https://doi.org/10.2196/jmir.6914

Aguilera, A., Schueller, S. M., \& Leykin, Y. (2015). Daily mood ratings via text message as a proxy for clinic based depression assessment. Journal of Affective Disorders, 175, 471-474. https://doi.org/10.1016/j.jad.2015 .01 .033

Alleman, J. R. (2002). Online counseling: The Internet and mental health treatment. Psychotherapy: Theory, Research, Practice, Training, 39(2), 199-209. https://doi.org/10.1037/0033-3204.39.2.199

American Psychiatric Association. (2013). Diagnostic and statistical manual of mental disorders (5th ed.).

Atkins, D. C., \& Christensen, A. (2001). Is professional training worth the bother? A review of the impact of psychotherapy training on client outcome. Australian Psychologist, 36(2), 122-130. https://doi.org/10 .1080/00050060108259644

Atun, R. A., \& Sittampalam, S. R. (2006). A review of the characteristics and benefits of SMS in delivering healthcare. The role of mobile phones in increasing accessibility and efficiency in healthcare report (pp. 18-28). Vodafone.

Baldwin, S. A., Berkeljon, A., Atkins, D. C., Olsen, J. A., \& Nielsen, S. L. (2009). Rates of change in naturalistic psychotherapy: Contrasting doseeffect and good-enough level models of change. Journal of Consulting and Clinical Psychology, 77(2), 203-211. https://doi.org/10.1037/a0015235

Bass, J. K., Annan, J., McIvor Murray, S., Kaysen, D., Griffiths, S., Cetinoglu, T., Wachter, K., Murray, L. K., \& Bolton, P. A. (2013). Controlled trial of psychotherapy for Congolese survivors of sexual violence. The New England Journal of Medicine, 368, 2182-2191. https://doi.org/10.1056/NEJMoa1211853

Bates, D., Maechler, M., Bolker, B., \& Walker, S. (2015). Fitting linear mixed-effects models using lme4. Journal of Statistical Software, 67(1), 1-48. https://doi.org/10.18637/jss.v067.i01

Baumel, A., Muench, F., Edan, S., \& Kane, J. M. (2019). Objective user engagement with mental health apps: Systematic search and panel-based usage analysis. Journal of Medical Internet Research, 21(9), e14567. https://doi.org/10.2196/14567

Blevins, C. A., Weathers, F. W., Davis, M. T., Witte, T. K., \& Domino, J. L. (2015). The posttraumatic stress disorder checklist for DSM-5 (PCL-5): Development and initial psychometric evaluation. Journal of Traumatic Stress, 28(6), 489-498. https://doi.org/10.1002/jts.22059

Bovin, M. J., Marx, B. P., Weathers, F. W., Gallagher, M. W., Rodriguez, P., Schnurr, P. P., \& Keane, T. M. (2016). Psychometric properties of the PTSD checklist for diagnostic and statistical manual of mental disordersfifth edition (PCL-5) in veterans. Psychological Assessment, 28(11), 1379-1391. https://doi.org/10.1037/pas0000254

Butollo, W., Karl, R., König, J., \& Rosner, R. (2016). A randomized controlled clinical trial of dialogical exposure therapy versus cognitive processing therapy for adult outpatients suffering from PTSD after type I 
trauma in adulthood. Psychotherapy and Psychosomatics, 85(1), 16-26. https://doi.org/10.1159/000440726

Cognitive Processing Therapy. (2020). https://cptforptsd.com/cpt-providerroster/

Cohen, J. (1977). Statistical power analysis for the behavioral sciences. Academic Press.

Dehejia, R. H., \& Wahba, S. (2002). Propensity score-matching methods for nonexperimental causal studies. The Review of Economics and Statistics, 84(1), 151-161. https://doi.org/10.1162/003465302317331982

Forbes, D., Lloyd, D., Nixon, R. D. V., Elliott, P., Varker, T., Perry, D., Bryant, R. A., \& Creamer, M. (2012). A multisite randomized controlled effectiveness trial of cognitive processing therapy for military-related posttraumatic stress disorder. Journal of Anxiety Disorders, 26(3), 442-452. https://doi.org/10.1016/j.janxdis.2012.01.006

Galovski, T. E., Blain, L. M., Mott, J. M., Elwood, L., \& Houle, T. (2012). Manualized therapy for PTSD: Flexing the structure of cognitive processing therapy. Journal of Consulting and Clinical Psychology, 80(6), 968981. https://doi.org/10.1037/a0030600

Ho, D., Imai, K., King, G., \& Stuart, E. (2011). MatchIt: Nonparametric preprocessing for parametric causal inference. Journal of Statistical Software, 42(8), 1-28. https://doi.org/10.18637/jss.v042.i08

Hoermann, S., McCabe, K. L., Milne, D. N., \& Calvo, R. A. (2017). Application of synchronous text-based dialogue systems in mental health interventions: Systematic review. Journal of Medical Internet Research, 19(8), e267. https://doi.org/10.2196/jmir.7023

Hollon, S. D., Muñoz, R. F., Barlow, D. H., Beardslee, W. R., Bell, C. C., Bernal, G., Clarke, G. N., Franciosi, L. P., Kazdin, A. E., Kohn, L., Linehan, M. M., Markowitz, J. C., Miklowitz, D. J., Persons, J. B., Niederehe, G., \& Sommers, D. (2002). Psychosocial intervention development for the prevention and treatment of depression: Promoting innovation and increasing access. Biological Psychiatry, 52(6), 610-630. https://doi.org/10.1016/S0006-3223(02)01384-7

Hull, T. D. \& Mahan, K. (2017). A study of asynchronous mobile-enabled SMS text psychotherapy. Telemedicine and eHealth, 23(3), 240-247. https://doi.org/10.1089/tmj.2016.0114

Hull, T. D., Malgaroli, M., Connolly, P. S., Feuerstein, S., \& Simon, N. M. (2020). Two-way messaging therapy for depression and anxiety: Longitudinal response trajectories. BMC Psychiatry, 20, Article 297. https:// doi.org/10.1186/s12888-020-02721-x

Jacobson, N. S., \& Truax, P. (1991). Clinical significance: A statistical approach to defining meaningful change in psychotherapy research. Journal of Consulting and Clinical Psychology, 59(1), 12-19. https:// doi.org/10.1037/0022-006X.59.1.12

Kirk, R. E. (1996). Practical significance: A concept whose time has come. Educational and Psychological Measurement, 56(5), 746-759. https:// doi.org/10.1177/0013164496056005002

Kroenke, K., \& Spitzer, R. L. (2002). The PHQ-9: A new depression diagnostic and severity measure. Psychiatric Annals, 32(9), 509-515. https://doi.org/10.3928/0048-5713-20020901-06

Kroenke, K., Spitzer, R. L., \& Williams, J. B. (2001). The PHQ-9: Validity of a brief depression severity measure. Journal of General Internal Medicine, 16(9), 606-613. https://doi.org/10.1046/j.1525-1497.2001.016009606.x

Kroenke, K., Strine, T. W., Spitzer, R. L., Williams, J. B., Berry, J. T., \& Mokdad, A. H. (2009). The PHQ-8 as a measure of current depression in the general population. Journal of Affective Disorders, 114(1-3), 163-173. https://doi.org/10.1016/j.jad.2008.06.026

Kuester, A., Niemeyer, H., \& Knaevelsrud, C. (2016). Internet-based interventions for posttraumatic stress: A meta-analysis of randomized controlled trials. Clinical Psychology Review, 43, 1-16. https://doi.org/10 .1016/j.cpr.2015.11.004

Kuhn, E., \& Owen, J. E. (2020). Advances in PTSD treatment delivery: The role of digital technology in PTSD treatment. Current Treatment Options in Psychiatry, 7, 88-102. https://doi.org/10.1007/s40501-02000207-x
Kuznetsova, A., Brockhoff, P., \& Christensen, R. (2017). lmerTest package: Tests in linear mixed effects models. Journal of Statistical Software, 82(13), 1-26. https://doi.org/10.18637/jss.v082.i13

Lewis, C., Roberts, N. P., Simon, N., Bethell, A., \& Bisson, J. I. (2019). Internet-delivered cognitive behavioural therapy for post-traumatic stress disorder: Systematic review and meta-analysis. Acta Psychiatrica Scandinavica, 140(6), 508-521. https://doi.org/10.1111/acps.13079

Lindhiem, O., Bennett, C. B., Rosen, D., \& Silk, J. (2015). Mobile technology boosts the effectiveness of psychotherapy and behavioral interventions: A meta-analysis. Behavior Modification, 39(6), 785-804. https://doi.org/10.1177/0145445515595198

LoSavio, S. T., Dillon, K. H., Murphy, R. A., Goetz, K., Houston, F., \& Resick, P. A. (2019). Using a learning collaborative model to disseminate cognitive processing therapy to community-based agencies. Behavior Therapy, 50(1), 36-49. https://doi.org/10.1016/j.beth.2018.03.007

Maguen, S., Li, Y., Madden, E., Seal, K. H., Neylan, T. C., Patterson, O. V., DuVall, S. L., Lujan, C., \& Shiner, B. (2019). Factors associated with completing evidence-based psychotherapy for PTSD among veterans in a national healthcare system. Psychiatry Research, 274, 112-128. https:// doi.org/10.1016/j.psychres.2019.02.027

Maieritsch, K. P., Smith, T. L., Hessinger, J. D., Ahearn, E. P., Eickhoff, J. C., \& Zhao, Q. (2016). Randomized controlled equivalence trial comparing videoconference and in person delivery of cognitive processing therapy for PTSD. Journal of Telemedicine and Telecare, 22(4), 238243. https://doi.org/10.1177/1357633X15596109

Malgaroli, M., Hull, T. D., Wiltsey Stirman, S., \& Resick, P. (2020). Message delivery for the treatment of posttraumatic stress disorder: Longitudinal observational study of symptom trajectories. Journal of Medical Internet Research, 22(4), e15587. https://doi.org/10.2196/15587

Marques, L., Valentine, S. E., Kaysen, D., Mackintosh, M. A., Dixon De Silva, L. E., Ahles, E. M., Youn, S. J., Shtasel, D. L., Simon, N. M., \& Wiltsey Stirman, S. (2019). Provider fidelity and modifications to cognitive processing therapy in a diverse community health clinic: Associations with clinical change. Journal of Consulting and Clinical Psychology, 87(4), 357-369. https://doi.org/10.1037/ccp0000384

Melville, K. M., Casey, L. M., \& Kavanagh, D. J. (2010). Dropout from internet-based treatment for psychological disorders. British Journal of Clinical Psychology, 49(4), 455-471. https://doi.org/10.1348/ 014466509X472138

Monson, C. M., Shields, N., Suvak, M. K., Lane, J. E., Shnaider, P., Landy, M. S. H., Wagner, A. C., Sijercic, I., Masina, T., Wanklyn, S. G., \& Wiltsey Stirman, S. (2018). A randomized controlled effectiveness trial of training strategies in cognitive processing therapy for posttraumatic stress disorder: Impact on patient outcomes. Behaviour Research and Therapy, 110, 31-40. https://doi.org/10.1016/j.brat.2018.08.007

Morland, L. A., Mackintosh, M. A., Rosen, C. S., Willis, E., Resick, P., Chard, K., \& Frueh, B. C. (2015). Telemedicine versus in-person delivery of cognitive processing therapy for women with posttraumatic stress disorder: A randomized noninferiority trial. Depression and Anxiety, 32(11), 811-820. https://doi.org/10.1002/da.22397

Nutting, P. A., Rost, K., Dickinson, M., Werner, J. J., Dickinson, P., Smith, J. L., \& Gallovic, B. (2002). Barriers to initiating depression treatment in primary care practice. Journal of General Internal Medicine, 17(2), 103-111. https://doi.org/10.1046/j.1525-1497.2002.10128.x

Oquendo, M. A., Halberstam, B., \& Mann, J. J. (2003). Risk factors for suicidal behavior: The utility and limitations of research instruments. In M. First (Ed.), Standardized evaluation in clinical practice (pp. 103-130). American Psychiatric Association.

R Core Team. (2018). R: A language and environment for statistical computing [Computer software]. R Foundation for Statistical Computing. https://www.R-project.org/

Resick, P. A., Galovski, T. E., Uhlmansiek, M. O. B., Scher, C. D., Clum, G. A., \& Young-Xu, Y. (2008). A randomized clinical trial to dismantle components of cognitive processing therapy for posttraumatic stress 
disorder in female victims of interpersonal violence. Journal of Consulting and Clinical Psychology, 76(2), 243-258. https://doi.org/10.1037/0022006X.76.2.243

Resick, P. A., Monson, C. M., \& Chard, K. M. (2016). Cognitive processing therapy for PTSD: A comprehensive manual. Guilford Press.

Resick, P. A., Wachen, J. S., Dondanville, K. A., Pruiksma, K. E., Yarvis, J. S., Peterson, A. L., Mintz, J., \& the STRONG STAR Consortium. (2017). Effect of group vs individual cognitive processing therapy in active-duty military seeking treatment for posttraumatic stress disorder: A randomized clinical trial. JAMA Psychiatry, 74(1), 28-36. https://doi.org/10.1001/ jamapsychiatry.2016.2729

Rice, R. E., \& Katz, J. E. (2003). Comparing internet and mobile phone usage: Digital divides of usage, adoption, and dropouts. Telecommunications Policy, 27, 597-623. https://doi.org/10.1016/S0308-5961(03) 00068-5

Roberts, A. L., Gilman, S. E., Breslau, J., Breslau, N., \& Koenen, K. C. (2011). Race/ethnic differences in exposure to traumatic events, development of post-traumatic stress disorder, and treatment-seeking for posttraumatic stress disorder in the United States. Psychological Medicine, 41(1), 71-83. https://doi.org/10.1017/S0033291710000401

Schulz, P. M., Resick, P. A., Huber, L. C., \& Griffin, M. G. (2006). The effectiveness of cognitive processing therapy for PTSD with refugees in a community setting. Cognitive and Behavioral Practice, 13(4), 322-331. https://doi.org/10.1016/j.cbpra.2006.04.011

Shin, C., Lee, S. H., Han, K. M., Yoon, H. K., \& Han, C. (2019). Comparison of the usefulness of the PHQ-8 and PHQ-9 for screening for major depressive disorder: Analysis of psychiatric outpatient data. Psychiatry Investigation, 16(4), 300-305. https://doi.org/10.30773/pi.2019.02.01

Szafranski, D. D., Smith, B. N., Gros, D. F., \& Resick, P. A. (2017). High rates of PTSD treatment dropout: A possible red herring? Journal of Anxiety Disorders, 47, 91-98. https://doi.org/10.1016/j.janxdis.2017 .01 .002

Titov, N., Dear, B. F., Staples, L. G., Bennett-Levy, J., Klein, B., Rapee, R. M., Andersson, G., Purtell, C., Bezuidenhout, G., \& Nielssen, O. B.
(2017). The first 30 months of the MindSpot Clinic: Evaluation of a national e-mental health service against project objectives. Australian and New Zealand Journal of Psychiatry, 51(12), 1227-1239. https://doi.org/ 10.1177/0004867416671598

Torous, J., Nicholas, J., Larsen, M. E., Firth, J., \& Christensen, H. (2018). Clinical review of user engagement with mental health smartphone apps: Evidence, theory and improvements. Evidence-Based Mental Health, 21(3), 116-119. https://doi.org/10.1136/eb-2018-102891

Wachen, J. S., Dondanville, K. A., Young-McCaughan, S., Mintz, J., LapizBluhm, M. D., Pruiksma, K. E., Yarvis, J. D., Peterson, A. L., Resick, P. A., \& the STRONG STAR Consortium. (2019). Testing a variable-length Cognitive Processing Therapy intervention for posttraumatic stress disorder in active duty military: Design and methodology of a clinical trial. Contemporary Clinical Trials Communications, 15, 100381. https:// doi.org/10.1016/j.conctc.2019.100381

Weathers, F. W., Litz, B. T., Keane, T. M., Palmieri, P. A., Marx, B. P., \& Schnurr, P. P. (2013a). The PTSD Checklist for DSM-5 (PCL-5). http:// www.ptsd.va.gov/

Weathers, F. W., Litz, B. T., Keane, T. M., Palmieri, P. A., Marx, B. P., \& Schnurr, P. P. (2013b). The PTSD Checklist for DSM-5 (PCL-5) - LEC-5 and Extended Criterion A. http://www.ptsd.va.gov/

Wilk, J. E., West, J. C., Duffy, F. F., Herrell, R. K., Rae, D. S., \& Hoge, C. W. (2013). Use of evidence-based treatment for posttraumatic stress disorder in Army behavioral healthcare. Psychiatry: Interpersonal and Biological Processes, 76(4), 336-348. https://doi.org/10.1521/psyc.2013.76.4.336

Young, K. S. (2005). An empirical examination of client attitudes towards online counseling. CyberPsychology \& Behavior, 8(2), 172-177. https:// doi.org/10.1089/cpb.2005.8.172

Received May 8, 2020

Revision received December 9, 2020

Accepted December 11, 2020 